\title{
Recent Trends, Applications, and Challenges of Brain-Computer Interfacing (BCI)
}

\author{
Aroosa Umair, Ureeba Ashfaq, and Muhammad Gufran Khan \\ Department of Electrical Engineering, National University of Computer and Emerging Sciences, Chiniot-Faisalabad, \\ 35400/38000, Pakistan \\ E-mail: aroosa.umair@nu.edu.pk,f159109@nu.edu.pk,m.gufran@nu.edu.pk
}

\begin{abstract}
Brain-Computer Interfacing (BCI) enables a communication pathway that is used to directly control certain object or device with the human brain. It is possible to acquire data from the brain with the help of sensors which essentially monitor the physical processes that occur in the brain and with the help of software we can direct a device accordingly. BCI introduces its users a new system that communicates in a special way without the use of muscles. BCI also provides a useful platform for the people with physical disabilities to conveniently perform certain tasks in our society. It uses brain imaging technologies which help in increasing the quality of the communication between humans and machines. There has been significant research effort in the past decade to explore different aspects of this promising field of technology. Previously, BCIs had limited functionality but due to recent advancement in technology it has attained the maturity of its own right by adding new trends and extra features to it.

In this paper, research work of development and integration of both hardware and software in BCI and the advancements of BCI are surveyed considering all the possibilities of direct communication of computers with a brain using emerging technologies. Different approaches used up till now with an overview of the methodology are presented to make the reader understand its meaning and functionality in a compact way. This research paper will guide a beginner to end up with a thorough knowledge of what's, how's and why's of BCI. In addition, BCI applications, challenges, possible solutions and future directions have also been discussed.
\end{abstract}

Index Terms-Brain Computer Interface, BCI, EEG, MMI.

\section{INTRODUCTION}

For generations, human's utmost desire is to conquer each and every part of this universe. He has left no stone unturned to find the secrets buried in this universe. The human brain is something very complicated and interesting to discover and the humans are very eager to explore it since long. During the early era of technology, neural activity remained a mystery to man, but now he has not only explored and understood the neural activities to a large extent but also utilized it to integrate with different systems. These efforts were made to interact human brain with the machines around us in order to make them functional with brain signals. From there the term "Brain-computer interfacing" came into existence.

The development of electroencephalography (EEG), which monitors and records the electrical activity of the human brain, laid the concept of BCI. Primarily driven by the human need to handle the physical disorders, many experiments based on neural plasticity were performed. As a result, it was proved that with the help of cortical plasticity, the prosthesis signals can be received by the brain as natural ones. After which the first prosthetic device was implanted in the mid of 1990s.

An illustration of brain-computer interfacing is shown in Fig. 1. Brain-computer interfacing (BCI) also well known by many other terms like mind-machine interfacing (MMI) and direct neural interfacing (DNI) has a lot of research work done under its trademark. According to Selim et al. [1], the main application of BCI is the providence of ability to a disabled person to communicate with the world around. In addition, there are many applications of BCI in the form of tools such as gaming for entertainment and exploring someone's brain to judge the mood swings and emotions which are the most advanced form up till now. So, we can say that BCI has very powerful impact in today's era and a further headway in science is impossible without it.

Many achievements have been made under the flag of this scientific expression in the areas of the prosthesis, environmental control, neural gaming and many others. Where there is some research there is a bundle full of literature. Many research papers have been published under this title to facilitate the readers. But none of them goes with an overall compact review. Some research papers handle the prosthetic side, some are related to the entertainment area and many deal with the environmental control side.

Keeping in view the importance of this field, a compact, well oriented and coherent material is necessary that presents the past, present and possible future work in this area. Our research paper discusses BCI during its early days in the 90's towards the advancement up till now and the future possibilities that will involve mentally controlling the physical activities. The survey is useful for beginners who would like to have a comprehensive overview of the field of BCI.

The remaining parts of the paper are organized as follows: Section II presents a short survey of related work. 
Section III includes BCI functionality, and classification. Section IV presents BCI applications in different areas of everyday life. Section $\mathrm{V}$ presents challenges faced in the field of BCI and finally, Section VI concludes the paper.

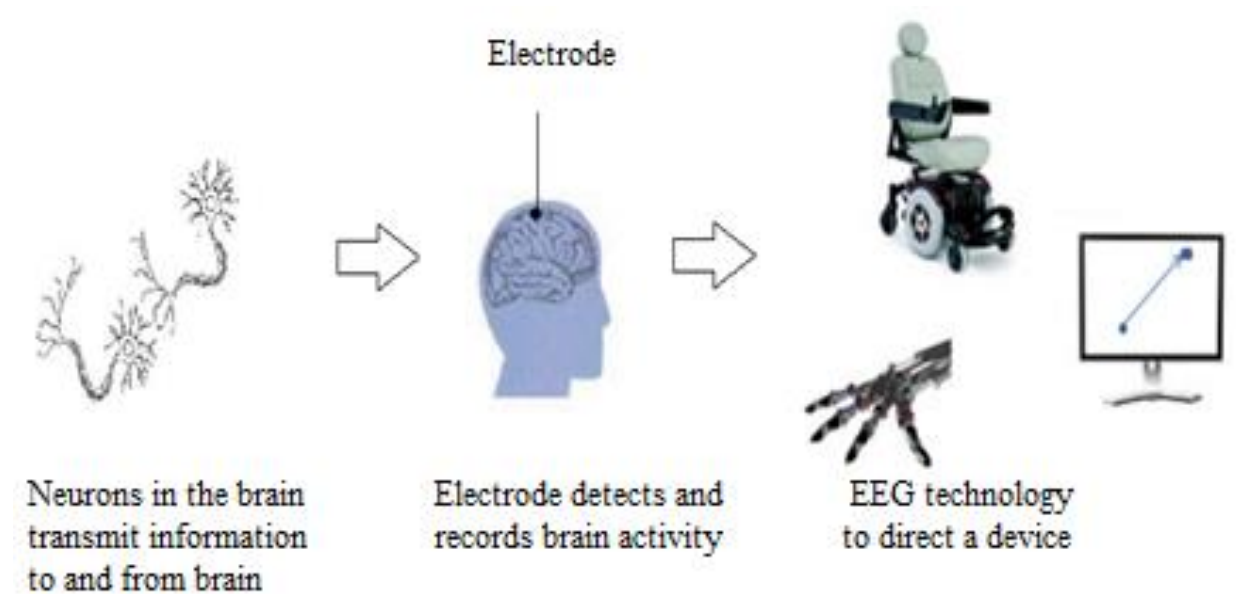

Fig.1. An illustration of Brain Computer Interfacing

\section{RELATED WORK}

In past authors have written many survey papers to conclude their work in this field.

In a survey paper in 2013 Ferreira et al. [2] have researched that in the field of neuroscience, biomedicine, automation and control engineering and computer science, research work started in the middle of 70s. . For their survey paper, they selected only recent paper published in the last five years. They have mentioned many challenges which are directly related to the effectiveness of interaction of Brain and Computer. They have also stated that in the near future BCIs will be more common as other interfaces like mobile devices and Kinect are today.

In another literature survey Hwang et al. [3] in 2013 provide us the number of published article of BCI in the year from 2007 to 2011 in the form of histograms as shown in Fig. 2. From their histograms presented in that paper we can say that the number of published articles is increasing per year.

In [4] Ibrahim has provided us a complete history of BCI work from 1929. In his survey paper, he concluded that BCI is the most popular research field in all over the world and it is the best solution for the treatment of lunatic and disabled people and also for the development of intelligence robots.

Blankertz et al. in [5] have discussed applications of BCI for rehabilitation and have mentioned challenges which are due to the illiteracy with BCI use and they believe that these issues will be overcome in near future.

Dhabale et al. in [6] have written literature survey on BCI controlled robot which is directly based on Brain Computer Interface. They are controlling robot using BCI with different techniques i.e. Bluetooth, pattern classifier technique and alpha brain waves etc.

\section{BCI FUNCTIONALITY AND CLASSIFICATION}

In this section, firstly a brief introduction to BCI is presented. Moreover, functionality and classification of $\mathrm{BCI}$ is discussed in the subsequent subsections.

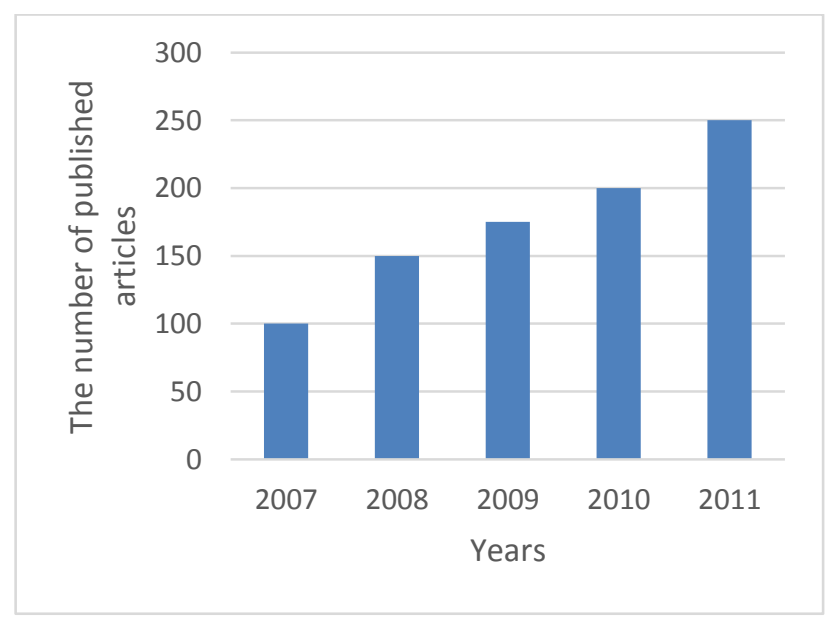

Fig.2. The no. of published BCI articles for each year from 2007 to 2011 [3].

\section{A. What is Brain-Computer Interface?}

BCI is a communication system between a brain and a computer in which these two parts mutually complement each other [7]. BCI is a non-muscular channel that creates understanding between users and their surroundings to help in communication between them. This interface enables communication with the help of user's mental activity without involving body's neuromuscular system.

\section{B. How a BCI functions?}

Our brain contains a complex network of around 100 billion neurons. When we think, move, feel or remember something these neurons work all the time and transmit information to and from the brain by producing electric 
signals. One common method is to detect those signals using "electroencephalography (EEG) technology" in which we use electrodes and record brain activity from the skull and use these electric signals to direct a device.

\section{Classification of BCI}

Brain-computer interfacing has the following two main types.

Invasive BCI: In this case, an array of electrodes is implanted in the gray matter of the patient's brain during a surgery. Electrodes are connected directly to neurons and thus every electrode records the electrical signals directly from the brain. Actual or imagined movements generate neuron spikes at different locations on the recording sites and with systematic training for a specific task neurons will fire in the same locations for different trials. Besides all this, invasive BCI causes inconvenience and risks to human health.

Non-invasive BCI: Non-invasive BCI systems capture good quality signals without any implantation in patient's brain. It can be categorized depending on what kind of imagery or mental tasks the user must perform in order to initiate or induce the command-related EEG response. In non-invasive BCI, as sensors are placed on the scalp, so it is easier to be used as compared to invasive technique.

\section{APPLICATIONS OF BRAIN-COMPUTER INTERFACING}

Brain-computer interfacing in its various forms has proven to have vast applications in almost every research field. The technology first introduced only to help the handicaps has now spread its roots enormously.

\section{A. Prosthetic Applications}

Vision: Invasive technologies were used to treat blindness for the very first time. Scientist William Do belle designed a working brain interface prototype implanted into a man blinded visual cortex in 1978 which contained 68 electrodes and produced the sensation of light without light entering the eye [8].This was the first public research which led the scientists, neurosurgeons
CPU manufacturers to think on these lines. At that time, it was a very young field of research. But after that many works has been done till now.

An important hardware was used by American scientists in a surgery in 2008, in which they inserted electro-biological parts and sensors inside the brain of a volunteer woman. She was blind by birth and volunteered her for this experiment to attach a camera toelectrobiological parts to replace the eye. This experiment finally worked after 48 hours surgery and 7 days post medication. She started watching and identifying everything but it was black $\mathrm{n}$ white, although, the camera was colored. After 2010 scientists started studying neuronetworks and their electro-biological interfaces with a computer in a more complex yet user-friendly way.

Movement: This side of $\mathrm{BCI}$ is used for individuals with paralysis to enable them to move their paralyzed muscles. This is usually done by providing them some special devices which assist the movement. These devices can be different types of interfaces with computers or even robot hands, arms, legs, knees wrists and other body parts for control.

Before the 20th century, wood hands were used as a replacement of any injury. But during the 20th century with the advancement in technology, scientists developed metallic arms that were capable of moving and were controlled by the brain. After the successful invention of the robotic arm, now scientists have developed a "MindControlled Prosthetic Robotic Arm" that can feel everything the person touches. This has proven a surprising invention by the US Defense Advanced Research Projects Agency (DARPA) in 2015. This prosthetic robotic arm is connected to the wearer's motor cortex and sensory cortex with the help of wires and as a result, it feels when a person touches something. The wires from the wearer's motor cortex control the motion and pressure of the robotic arm and that connect back into the sensory cortex which identifies the sensation that they are touching something [9]. A block diagram of the mind controlled prosthetic robotic arm is shown in Fig. 3.

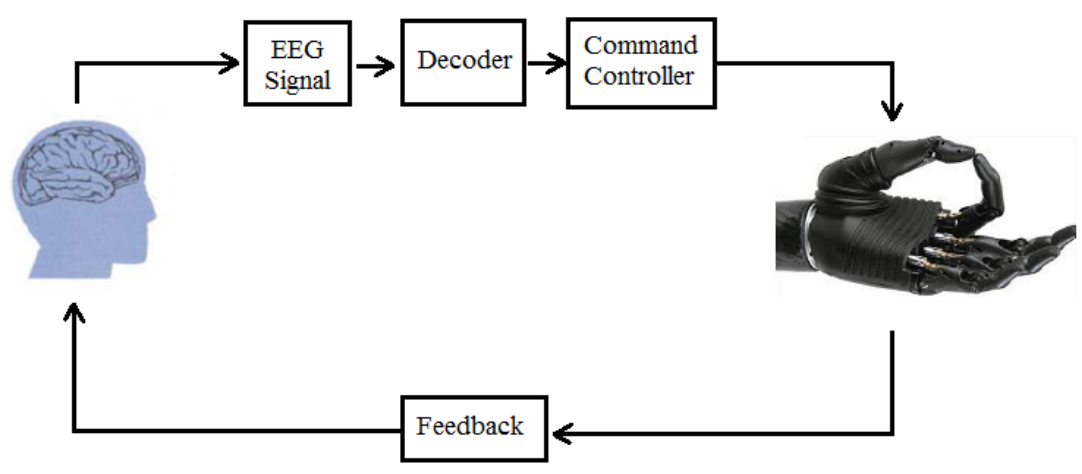

Fig.3. The mind controlled prosthetic robotic arm [9].

"Brain Gate", as shown in Fig. 4, is another prosthetic invention by the biotech company Cyber kinetics in the
Department of Neuroscience at Brown University. Actually, Brain Gate helps those people who cannot 
move or have lost sense to make any movement which includes persons with spinal cord injury, brainstem stroke or amyotrophic lateral sclerosis (ALS) [8].

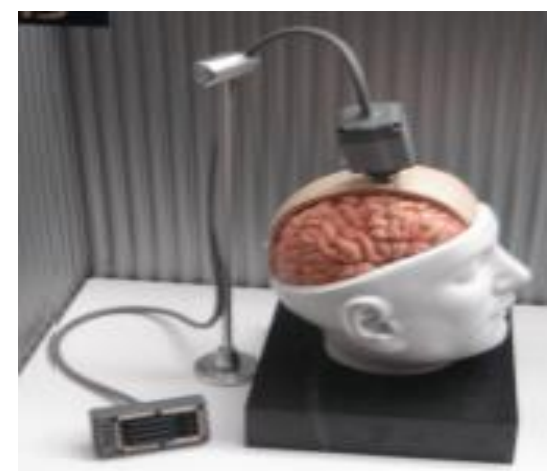

Fig.4. Brain Gate [8].

Voice: A voice's prosthesis is a silicon device which helps those patients who have speaking problems. During voice's prosthesis, voice box is replaced by the prosthetic device at the separation point of the windpipe and the food pipe. That separation point is called "Opening" [8]. In these voice prosthetic devices, there is a "Brain Reading Computer Software" which can read the EEG signals coming from the brain and send it to decoder who translates thoughts and generates respective voice against that code. A voice prosthetic must be changed after certain time otherwise it will cause a cough or some problem when any food item will stick in the windpipe. One other solution of this problem is that there are special plugs which can be used at the time of eating or drink to block any food item to enter the windpipe. The sound producing voice prostheses are shown in Fig.5.

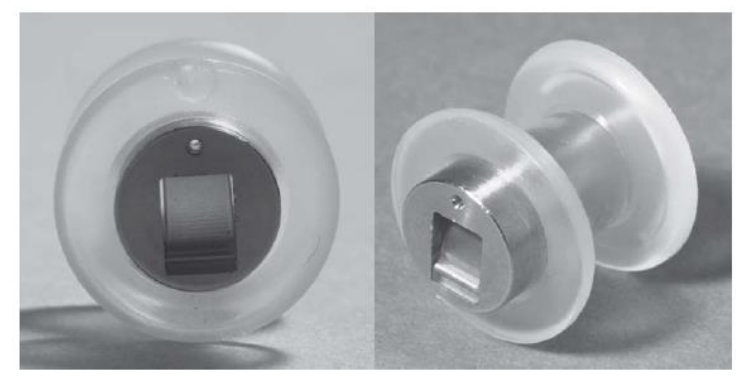

Fig.5. The sound producing voice prostheses [10].

In 1972, for the first time, an article by Mozolewski was described for prosthesis devices for voice rehabilitation in which these devices were categorized into two types; non-indwelling and indwelling voice prostheses [8]. The difference in both the devices is that non-indwelling voice prostheses are easily removable, cleanable and replaceable by the patients themselves while for indwelling prostheses, there must be a need of a medical expert. After that in 1980, the first commercially non-indwelling prosthetic device was introduced by Singer and Blom [8]. In 1984, the first indwelling voice prosthesis was described and after that many voice prosthesis were manufactured and were introduced to the market by Atos Medical and InHealth. "Provox NID" is a non-indwelling voice prosthetic device which can satisfy a patient and is feasible to him more than a device which is fixed inside him [11]. According to Hilgers et al. [12], they performed the experiment of prosthetic rehabilitation of voice on 79 patients and observed from the results that voice is much better in $91 \%$ of patients. According to Hilgers et al. [13], a new valve mechanism was introduced in voice prostheses which prevent the accidental opening of the valve and increases the voice prosthetic life time. Many experiments have been done for the improvement in voice prostheses by time and Provox ${ }^{\circledR} 2$ was introduced, in which discomfort of patients was reduced [14]. After Provox ${ }^{\circledR} 2$, new voice prosthetic device Vega 22.5 was introduced which was comparable to Provox ${ }^{\circledR} 2$ but with better voice characteristics [15]. Hilgers and Ward et al. [16] and [17] respectively focused on design improvement of voice prosthetic devices.

\section{B. Entertainment Applications}

In the entertainment field, Brain-computer interfacing has emerged as cutting edge technology in the form of neuro-gaming. This kind of gaming uses player's physiological functions like brain signals, player's heartbeat and facial expressions instead of traditional user controllers to control the game. It is only possible with the help of EEG headsets which record electrical signals in the brain. The earliest neuro-games include "Throw trucks with your mind", "Mind Balance video game", "NeuroRacer" and "NeuroMage". In all these games a user can get a good score by controlling a virtual character either by imagining movements, balancing weights or picking and throwing ability. For Mind Balance video game, see Fig. 6.

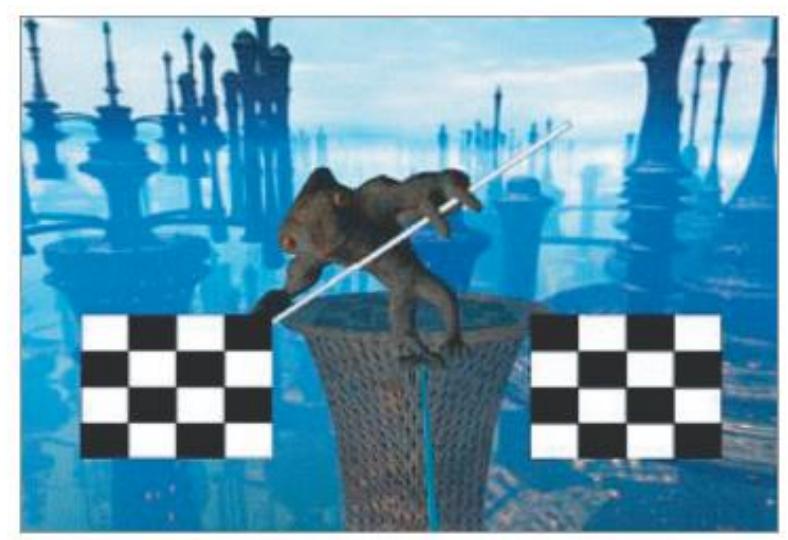

Fig. 6. Mind Balance video game [18].

\section{Safety and Security}

There are many applications in this area; the detection of irregular behavior and suspicious objects, detection of signal distortions with the help of EEG and eye movement [19]. In a scenario, many testers and observers are watching the recording of a suspicious event, only EEG, and precise eye movement can identify the potential targets that cannot be identified by any other way [19]. 
There are also some other interesting applications in this area such as lie detection using EEG which records physiological functions to detect a lie; it is very helpful in crime cases.

After 2010, many defense institutes and research centers associated with defense systems in many countries started working on BCI and are trying to download RAM, ROM and hard drives of brains. They claim these research works will be fruitful up to 2025 for criminal investigation, pharmaceutical companies, and a better understanding of the human body.

\section{User Attention System}

This kind of system was first introduced by Toyota in 2006 as user attention system as shown in Fig. 7. It uses infrared rays to examine the attentiveness of driver. The system is installed on the steering side of the vehicle that uses a CCD camera for eye tracking with the help of infrared rays. In the case of any ambiguity, the system alerts the driver by blinking the lights or creating sounds. In further cases, the vehicle will apply the brakes to stop the car [20]. In 2008, this system was further enhanced by Toyota to check the drowsiness of the driver.

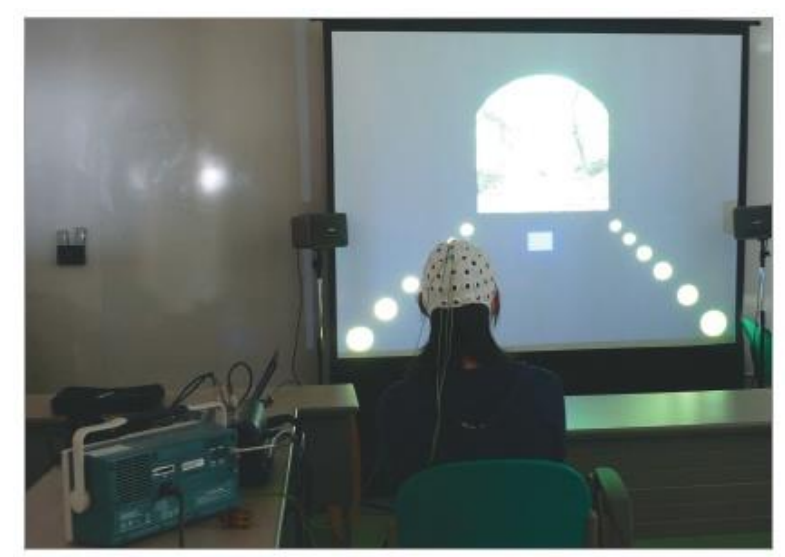

Fig.7. To monitor driver's alertness level in a virtual reality driving [18].

Further advancement in DCM includes a device that is now worn by the driver of the vehicle during driving. This device monitors the each and every state of the driver that includes drowsiness, anger, heartbeat, appetite and various other ones. It consists of a display unit, an information collection and a controller unit designed to collect information related to the user's body state and to sense a situation related to it respectively. After information collecting, it converts it into a numerical value that represents the body state of the user with respect to the sensed situation and accordingly performs the desired task.

\section{E. Communication}

Scientists assume that one day we will be able to directly connect with satellite and will see the world in an eye blink. This research can go beyond artificial intelligence but there is a risk of humans being more dependent towards machines, electronics, and networks.
There are also consequences of a total collapse in case of electro-biological virus penetration in the human body. It can be fatal and total loss. Although in this case, human power will touch the skies. Maybe a time will come when we will not depend on today's cellular network and will communicate thousands of miles away without using any external device. Our phone book will be embedded in our brain, and communication will be a "thought" away from us. Definitely, there would be a medium of communication but it will be entirely a different network and hardware.

Recently, Samsung is working to make an interface with the help of which tablets can be controlled through mind. This can indeed be very exciting news for frequent social media users [21].

\section{F. Biomedical}

In 2016 Kang et al. have researched that in field of electronic implants, a revolutionary invention is "Bioresorbable Chip" made up of silicon or any other non-harmful material. These chips are inserted to measure the damaged areas of brain, usually after stroke, by measuring the temperature and pressure inside. After a particular time period these chips dissolve automatically causing no harm to the patient [22].

Study of neural connection is the latest trend among the researchers these days. By making a slight change in neuronal communication network, behaviors can be changed. Human brain consists of $10^{11}$ neurons, thus making the neural connectivity very complex to answer that how the system communicate and control behaviors. Researchers at University of Massachusetts medical school thus switched their research on Nematode with 302 neurons, the only animal for which the complete neural road map has been defined. The study revealed that flipping the sign of a synapse from inhibitory to excitatory in the worms brain the behavior can be reversed. For this purpose they analyzed nematode touch response. During this response neurotransmitters are released that activate an inhibitory ion channel. This causes the worm to relax its head and quickly reverse direction away from the predator. This results in flipping the behavior and can be further used to make new neural circuits in coming future [23].

Now in connection to this neural study, the question was can our brains be reprogrammed to improve many short comings most of the people are facing. If it is so, how a brain can be reprogramed? In answer to this, the researchers at University of Western Australia in collaboration with other students have shown that abnormal neural connections can be changed to normal ones with the help of weak electromagnetic signals. This experiment was performed on mice and can may have different effects on humans. The research is still under process that how human minds can be reprogramed by this study and what would be the effects. University of Western Australia has concluded that once they are succeeded in their research, they can lead to cure depression, epilepsy tinnitus and weakness of brain [24]. 


\section{Challenges FACEd IN The FIELd OF BCI}

Every achievement is followed by many challenges indeed. Scientists face many hurdles in overcoming the ambiguities while designing something amazing and new. Brain-computerinterfacing, though, not something new to us, is even now followed by many challenges.

\section{A. Understanding Human Brain Activity}

For a non-medical person, especially an engineer, understanding the human brain behavior is a very difficult task indeed. While developing an interfaced device the person needs to understand the brain functionality which is not an easy job. The person first has to identify the key areas that should be focused on while working on a prosthetic or any other BCI device which is a very challenging task.

\section{B. Usability}

Most of the time the user wants the system to be easily approachable. A Very complex system that is not easy to handle is usually disliked. To make such a complex system user-friendly is a very difficult task. Conventional EEG process includes the application of gel to the electrodes to measure the exact level of volt potential. In contrary to this, a new EEG technique has been introduced that does not need the application of gel in any case and provide $70 \%$ accurate results [5]. Furthermore, the system must be safe and secure.

\section{Capturing Minute Details}

Keeping in view the complexity of the system, the BCI device has to capture each and every movement it is designed for. Like in DSM if the eye detection system fails to detect the eyes when the person is depending on it, he will undergo through a fatal accident.

\section{Wearing Problems}

While wearing a prosthetic device a common problem of sweating arises. Moreover, large energy consumption when a person puts on such a device is also a challenge.

\section{E. Hardware Challenge}

Many hardware improvements are required to make the system work outside the laboratories and sensitive areas. Such systems should be smart enough to work outside the suitable environment like at noisy places. They must have designed in order to distinguish between the actual input and the random ones in such places.

\section{F. Ethical Challenge}

While discussing the Ethical issues of BCI, there comes a word cyborg in which there exists a concept of enhancement of physical abilities of a normal human being. A cybernetic human will be more powerful than a normal one when he comes in a competition. These kinds of uses will undermine the usefulness of BCI and will enhance cheating. Second thing is that a headset with greater range of brain signal detection may read the mind of others; this is also ethically not good.

\section{G. Small training set}

Human brain is very complex, non-linear and nonstationary system which is actually a high challenge among all the challenges faced for BCI. Since the training process is affected by all the previous challenges i.e. understanding, usability issues, capturing minute details, wearing problems and hardware challenge, so it integrates a big challenge. Although too much training is time consuming but still the training sets in this case are relatively small in training BCI interfaces.

\section{CONCLUSION}

Brain-computer interface is indeed a milestone in the history of science which provides a channel's facility between brain and device. This paper presented a study of BCI with complete knowledge of its working along with the challenges and possible solutions which identify future directions and encourage BCI researchers around the world. In our research it is found that EEG is predominant technology due to its advantages, for example it can direct any prosthetic device for any kind of paralyzed patient for vision, movement, and voice disability just in five stages. These stages are signal acquisition, preprocessing and signal enhancement, feature extraction, feature classification and finally the control interface. It can also help in capturing the brain signal for applications such as monitoring, safety, security, entertainment, medical, transportation and authentication. The study of BCI applications shows that the user can do any kind of work which is difficult or impossible for a paralyzed person, with the help of only his/her thoughts and without involving any paralyzed human organ. But there are still few challenges that must be resolved to enhance the technology of BCI, for example understanding human brain activity, usability, capturing minute details, wearing problems, hardware problems and ethical issues.

Hence, based on the study presented in this paper, there is no hesitation in acknowledging that after a decade or so, when these challenges would also be resolved, we will be able to control the power of decision making by collecting data from five senses and manipulating human body to act and react to certain situations in an entirely different way. We will be soon in a state of removing the bad impressions, ideas and activities from our body by reprogramming our brains and thus resolving the psychological problems that are a cause of increasing depression among humans. In near future applications of BCI will be used by a common man in his routine activities as we use computers, mobile phones, and many other technologies today. In the more distant future, developments in BCI will have vast consequences for the interaction between human beings. Concisely, we are advancing towards connecting the crowd through neural signals.

\section{REFERENCES}

[1] A. E. Selim, M. A. Wahed and Y. M. Kadah, "Machine 
Learning Methodologies in Brain-Computer Interface Systems," in Proc. Cairo Int. Biomedical Engineering Conf, 2008.

[2] A. L. S. Ferreira, L. C. de Miranda, E. E. C. de Miranda and S. G. Sakamoto, "A survey of interactive systems based on brain-computer interfaces," SBC Journal on Interactive Systems, vol. 4, no. 1, pp. 3-13, 2013.

[3] H.-J. Hwang, S. Kim, S. Choi and C.-H. Im, "EEG-based brain-computer interfaces: a thorough literature survey," International Journal of Human-Computer Interaction, vol. 29, no. 12, pp. 814-826, 2013.

[4] I. Arafat, "Brain-Computer Interface: Past, Present \& Future," International Islamic University Chittagong (IIUC), Chittagong, Bangladesh, 2013.

[5] B. Blankertz, F. Popescu, M. Krauledat, S. Fazli, M. Tangermann, K. Müller, R. Grinter, T. Rodden, P. Aoki, E. Cutrell and others, "Challenges for brain-computer interface research for human-computer interaction applications," in ACM CHI Workshop on Brain-Computer Interfaces for HCI and Games, 2008.

[6] M. I. S. S. R. U. T. U. J. A. DHABALE, "LITERATURE SURVEY ON BCI CONROLLED ROBOT".

[7] J. R. Wolpaw, N. Birbaumer, D. J. McFarland, G. Pfurtscheller and T. M. Vaughan, "Brain--computer interfaces for communication and control," Clinical neurophysiology, vol. 113, no. 6, pp. 767-791, 2002.

[8] C. Postelnicu, D. Talaba and M. Toma, "Brain computer interfaces for medical applications," Bulletin of the Transilvania University of Bra Vol, vol. 3, p. 52, 2010.

[9] C. Ho, "Neuroprostheses," 2016.

[10] M. Van der Torn, A sound-producing voice prosthesis, $\mathrm{Ph}$. D. Thesis. Amsterdam. VU Medical Center: VU Medical Center, 2005.

[11] K. Hancock, B. Houghton, C. J. Van As-Brooks and W. Coman, "First clinical experience with a new nonindwelling voice prosthesis (Provox ${ }^{\circledR}$ NID $^{\mathrm{TM}}$ ) for voice rehabilitation after total laryngectomy," Acta otolaryngologica, vol. 125, no. 9, pp. 981-990, 2005.

[12] F. J. M. Hilgers and P. F. Schouwenburg, "A new lowresistance, self-retaining prosthesis (Provox ${ }^{\mathrm{TM}}$ ) for voice rehabilitation after total laryngectomy," The Laryngoscope, vol. 100, no. 11, pp. 1202-1207, 1990.

[13] F. J. M. Hilgers, A. H. Ackerstaff, A. J. M. Balm, M. W. M. Van Den Brekel, I. Bing Tan and J.-o. Persson, "A new problem-solving indwelling voice prosthesis, eliminating the need for frequent Candida-and "underpressure"-related replacements: Provox ActiValve," Acta oto-laryngologica, vol. 123, no. 8, pp. 972-979, 2003.

[14] F. J. M. Hilgers, A. H. Ackerstaff, A. J. M. Balm, I. B. Tan, N. K. Aaronson and J.-O. Persson, "Development and clinical evaluation of a second-generation voice prosthesis (Provox® 2), designed for anterograde and retrograde insertion," Acta oto-laryngologica, vol. 117, no. 6, pp. 889-896, 1997.

[15] F. J. M. Hilgers, A. H. Ackerstaff, I. Jacobi, A. J. M. Balm, I. B. Tan and M. W. M. van den Brekel, "Prospective clinical phase II study of two new indwelling voice prostheses (Provox Vega 22.5 and $20 \mathrm{Fr}$ ) and a novel anterograde insertion device (Provox Smart Inserter)," The Laryngoscope, vol. 120, no. 6, pp. 1135-1143, 2010.

[16] F. J. M. Hilgers, A. H. Ackerstaff, M. van Rossum, I. Jacobi, A. J. M. Balm, I. B. Tan and M. W. M. van den Brekel, "Clinical phase I/feasibility study of the next generation indwelling Provox voice prosthesis (Provox Vega)," Acta oto-laryngologica, vol. 130, no. 4, pp. 511$519,2010$.

[17] E. C. Ward, K. Hancock, N. Lawson and C. J. van As-
Brooks, "Perceptual characteristics of tracheoesophageal speech production using the new indwelling Provox Vega voice prosthesis: a randomized controlled crossover trial," Head \& neck, vol. 33, no. 1, pp. 13-19, 2011.

[18] A. Lécuyer, F. Lotte, R. B. Reilly, R. Leeb, M. Hirose, M. Slater and others, "Brain-computer interfaces, virtual reality, and videogames.," IEEE Computer, vol. 41, no. 10, pp. 66-72, 2008.

[19] S. Mathan, "Feature-Image search at the speed of thought.," interactions, vol. 15, no. 4, pp. 76-77, 2008.

[20] T. Brandt, R. Stemmer and A. Rakotonirainy, "Affordable visual driver monitoring system for fatigue and monotony," in Systems, Man and Cybernetics, 2004 IEEE International Conference on, 2004.

[21] S. Young, "Samsung demos a tablet controlled by your brain," technologyreview. com, 2013.

[22] S.-K. Kang, R. K. J. Murphy, S.-W. Hwang, S. M. Lee, D. V. Harburg, N. A. Krueger, J. Shin, P. Gamble, H. Cheng, $\mathrm{S}$. Yu and others, "Bioresorbable silicon electronic sensors for the brain," Nature, vol. 530, no. 7588, pp. 71-76, 2016.

[23] J. K. Pirri, D. Rayes and M. J. Alkema, "A Change in the Ion Selectivity of Ligand-Gated Ion Channels Provides a Mechanism to Switch Behavior," PLoS Biol, vol. 13, no. 9, p. e1002238, 2015.

[24] U. of Western Australia., "Could your brain be reprogrammed to work better?," ScienceDaily, ScienceDaily, 6 August 2014.

\section{Authors' Profiles}

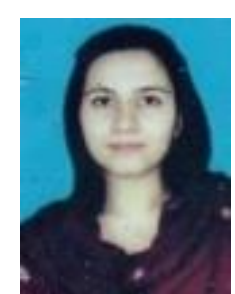

Aroosa Umair is working as a Lab Engineer at Department of Electrical Engineering, National University of Computer and Emerging Sciences, ChiniotFaisalabad, Pakistan. She is responsible for teaching and conducting Lab sessions of different Electrical Engineering and Computer Science courses. She is also doing her M.S. Electrical Engineering from School of Electrical Engineering FAST National University of Computer and Emerging Sciences CFD campus. Before that, she completed her B.S. Electrical Engineering from Lahore campus of FAST NUCES in 2014. She has been involved in different projects in the areas of Robotics and DSP. Her research interests include signal processing, machine learning, human-machine interfacing and robotics and data science.

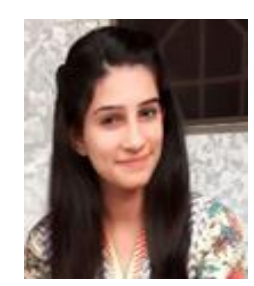

Ureeba Ashfaq is currently working as an Assistant Engineer in GC University, Faisalabad, Pakistan with responsibilities of handling the electrical engineering aspects of the on-going projects. She is doing her MS in Electrical Engineering from National University of Computer and Emerging Sciences (NUCES). She has completed her BS Electrical Engineering from University of Engineering \& Technology, Lahore with a final year project of "standalone photovoltaic system with dual axis tracker". She also led many projects like fiber-based IP system, information technology data center, hybrid, and grid tied based solar system. Here recent research interests in the field of BCI and cyberphysical systems. 


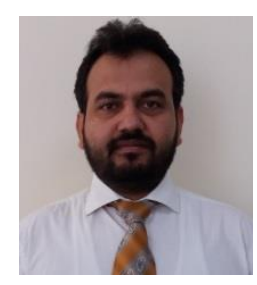

Muhammad Gufran Khan is currently working as an Assistant Professor at National University of Computer \& Emerging Sciences (NUCES), ChiniotFaisalabad, Pakistan. Before joining NUCES, he worked as an Analysis Engineer for automotive electronic and control systems at Volvo Corporation, Sweden. He has received his M.Sc. Electrical Engineering degree with an emphasis on Signal Processing in 2005 and the Ph.D. in 2011, both from Blekinge Institute of Technology, Sweden.

His current research interests are in Model-based system design, Cyber-physical systems, and Machine learning.

How to cite this paper: Aroosa Umair, Ureeba Ashfaq, Muhammad Gufran Khan,"Recent Trends, Applications, and Challenges of Brain-Computer Interfacing (BCI)", International Journal of Intelligent Systems and Applications(IJISA), Vol.9, No.2, pp.58-65, 2017. DOI: 10.5815/ijisa.2017.02.08 